\title{
Avaliação de extratos de plantas sobre a resposta imune, o desempenho produtivo e a morfometria intestinal de frangos de corte desafiados com Eimeria sp.
}

\author{
Assessment of plant extracts on immune response, productive performance and \\ intestinal morphometry of broilers challenged with Eimeria sp.
}

\author{
FERNANDES, Jovanir Inês Müller ${ }^{1 *}$; KOSMANN, Raquel Cristina ${ }^{1}$; VIOTT, Aline de \\ Marco $^{1}$; SIMÕES, Ricardo Scherer ${ }^{1}$, RIBEIRO, Mayra Vissotto ${ }^{1}$; RORIG, Anete $^{1}$
}

\author{
${ }^{1}$ Universidade Federal do Paraná, Programa de Pós-Graduação em Ciência Animal, Palotina, Paraná, Brasil. \\ *Endereço para correspondência: jimfernandes@ufpr.br
}

\section{RESUMO}

O objetivo foi avaliar o efeito do uso de extrato de plantas sobre a resposta imune, o desempenho produtivo e a morfometria intestinal de frangos de corte desafiados com a vacina contra coccidiose. Foram utilizados 720 pintos de corte, machos, distribuídos em um delineamento inteiramente casualizado com quatro tratamentos e nove repetições de 20 aves cada. A partir dos 14 dias, as aves passaram a receber os tratamentos experimentais compostos por A - Dieta basal + Antibiótico promotor do crescimento (APC) ; B Dieta basal + APC + desafio vacinal; $\mathrm{C}$ - Dieta basal + extrato de plantas, e D - Dieta basal + extrato de plantas + desafio vacinal. As aves dos tratamentos B e D receberam vacina oral contra coccidiose (vinte vezes a dose individual recomendada pelo fabricante). A adição de extrato de plantas à dieta de aves desafiadas com oocistos de Eimeria $s p$ aumentou $(\mathrm{p}<0,05)$ a secreção de IgA pela mucosa intestinal. As lesões microscópicas da mucosa intestinal não foram influenciadas $(p>0,05)$ pelo desafio ou pelo uso do extrato de plantas. O desafio com oocistos de Eimeria $s p$ ou associado ao extrato de plantas resultou em menor $(p<0,05)$ ganho de peso e pior conversão alimentar apenas na semana seguinte à inoculação. Os resultados obtidos mostraram que a adição de extrato de plantas à dieta de aves submetidas ao desafio vacinal aumentou a secreção de IgA pela mucosa intestinal e que a inclusão do extrato de plantas ou de melhorador de desempenho resultou em desempenho produtivo semelhante.

Palavras-chaves: coccidiose, ganho de peso, IgA, mucosa intestinal

\section{SUMMARY}

This study evaluated the effect of using plant extracts on immune response, productive performance, and intestinal morphometry of broilers challenged with coccidiosis vaccine. It was used 720 male Ross broiler chicks, distributed into a completely randomized design with four treatments and nine repetitions of 20 birds each. From the $14^{\text {th }}$ day, animals received the following treatments: A - Basal diet + antibiotic growth promoter (AGP); B - Basal diet $+\mathrm{AGP}+$ vaccine challenge; $\mathrm{C}$ - Basal diet + plant extract and D - Basal diet + plant extract + vaccine challenge. Broilers of treatments $B$ and $\mathrm{D}$ received oral coccidiosis vaccine (twenty times the label-recommended individual commercial dose). Adding plant extract to the diet of birds challenged with Eimeria $\mathrm{sp}$ increased $(\mathrm{p}<0.05)$ IgA secretion by the intestinal mucosa. Microscopic lesions of the intestinal mucosa were not affected $(p>0.05)$ by the immune challenge or addition of the plant extract. The challenge with oocysts of Eimeria $\mathrm{sp}$ or associated with the plant extract resulted in a smaller $(p<0.05)$ weight gain and worse feed conversion only in the next week of the inoculation. The results showed that the addition of plant extracts to the diet of birds submitted to vaccine challenged increased the secretion of $\operatorname{Ig} \mathrm{A}$ by the intestinal mucosa and that the inclusion of the plant extract or of the antibiotic growth promoter resulted in similar productive performance at the age of slaughter.

Keywords: coccidiosis, IgA, intestinal mucosa, weight gain 


\section{INTRODUÇÃO}

As doenças entéricas representam um dos maiores desafios para a avicultura industrial mundial nos últimos anos devido à perda de produtividade, ao aumento de mortalidade e à contaminação de produtos de origem avícola para o consumo humano. Por outro lado, é fato crescente a restrição, em todo o mundo, ao uso de antibióticos como aditivos na nutrição animal. A Comissão Europeia proibiu o uso desde 2006 (Regulamento CE N. 1831/2 003) (HUYGHEBAERT et al., 2011), o que têm impulsionado a pesquisa por alternativas que garantam $o$ máximo crescimento dos animais sem afetar a qualidade do produto final.

As principais alternativas que têm sido pesquisadas incluem prebióticos, enzimas, ácidos orgânicos e extratos vegetais (JEAUROUND et al., 2002; BONA et al., 2012), e são de interesse dos consumidores porque preferem aditivos naturais aos compostos sintéticos (PEARCE \& JIN, 2010). Na visão atual do consumidor, o conceito de qualidade do alimento engloba não só as características de sabor, aroma, aparência e padronização, mas também a preocupação em adquirir alimentos que não causem danos à saúde e ao meio ambiente. Têm-se questionado também a procedência e a forma com que os animais estão sendo produzidos.

Os extratos vegetais são substâncias derivadas de plantas medicinais ou de especiarias (óleo essencial, extrato vegetal, oleoresina) e podem atuar inibindo $o$ crescimento de microrganismos patogênicos no intestino (APPLEGATE et al., 2010), melhorando a digestibilidade dos nutrientes, estimulando a secreção intestinal de muco (JAMROZ et al., 2006) devido as diversas características como atividade antimicrobiana, antioxidante, e imunomoduladora (FUKAYAMA et al., 2005; TOLEDO et al., 2007; RIZZO et al., 2010).

Os protozoários de gênero Eimeria sp., se multiplicam nas células do intestino e provocam dano tecidual, que é causa de interrupção do consumo e dos processos digestivos e absortivos e da predisposição para o desenvolvimento das enterites. Surtos de coccidiose normalmente antecedem ou ocorrem concomitantemente com achados de lesões causadas pelo gênero Clostridium sp. (McDEVITT et al., 2006), uma vez que os danos causados na mucosa facilitam o estabelecimento e a multiplicação deste agente.

O objetivo foi avaliar o efeito da inclusão de extrato de plantas sobre o título da imunoglubulina IgA, lesões microscópicas e morfometria da mucosa intestinal e desempenho zootécnico de frangos de corte submetidos ao desafio entérico com oocistos de Eimeria sp.

\section{MATERIAL E METODOS}

O experimento foi conduzido no aviário experimental da Universidade Federal do Paraná - Campus Palotina, Palotina-PR. O protocolo experimental foi realizado em conformidade com os procedimentos descritos no protocolo n. 13/2010 e aprovado pelo Comitê de Ética para o Uso de Animais (CEUA/ UFPR).

Foram utilizados 720 pintos de corte, de 1 dia de idade, machos, linhagem Ross, provenientes de matrizes de cerca de 40 semanas. No alojamento, os pintos foram alocados aleatoriamente de acordo com um delineamento inteiramente casualizado com quatro tratamentos e nove repetições de 20 aves cada, compondo 36 unidades experimentais. De 1 a 14 dias de idade todas as aves receberam ração basal. A partir dos 14 
dias passaram a receber os tratamentos experimentais compostos por A: - Dieta basal + Antibiótico promotor do crescimento (APC); B - Dieta basal + $\mathrm{APC}+$ desafio vacinal; $\mathrm{C}$ - Dieta basal + extrato de plantas, e D - Dieta basal + extrato de plantas + desafio vacinal.

O protocolo de desafio vacinal com Eimeria (utilizando 20 vezes a dose recomendada pelo fabricante de uma vacina comercial contra coccidiose) foi aplicado nas aves dos tratamentos B e D. A vacina utilizada oocistos vivos atenuados das espécies E. acervulina, $E$. máxima, E. praecox, E. tenella e E. mitis. Previamente à aplicação foi realizada a esporulação dos oocistos pela injeção de $\mathrm{O}_{2}$ diretamente em tubos Falcon com a vacina, os quais foram rapidamente lacrados e mantidos em estufa BOD a $28^{\circ} \mathrm{C}$ durante 48 horas. A vacina foi inoculada diretamente no ingluvio de cada ave ( \pm 80.000 oocistos).

A dieta basal foi suplementada com antibiótico promotor de crescimento a base de enramicina, na dose de $125 \mathrm{gr} / \mathrm{ton}$ de ração (Enramax ${ }^{\circledR}$ - Farmabase Saúde Animal LTDA) ou com extrato de plantas, na dose de $1,5 \mathrm{Kg} /$ ton de ração do produto comercial (Essential ${ }^{\circledR}$ Oligobasics), contendo o extrato de mamona e de caju. A mamona (Ricinus communis $L$.) contém $90 \%$ de ácido graxo ricinoléico, enquanto que o óleo obtido da castanha do caju (Anacardium occidentale) possui como princípios ativos o ácido anacárdico $(2-3 \%)$, cardol $(15-18 \%)$ e cardanol $(75-80 \%)$. Água e ração foram fornecidas à vontade em um programa de alimentação, dividido em duas fases: a inicial, de 1 a 14 dias de idade e a de crescimento/abate, de 15 a 42 dias de idade de acordo com as recomendações nutricionais da linhagem (Tabela 01).

Os parâmetros de desempenho (peso vivo, consumo de ração e conversão alimentar) foram registrados aos 14, 21, 28 e 42 dias de idade por parcela experimental de 20 aves. $O$ peso vivo das aves foi obtido separadamente das aves desafiadas das não desafiadas. Aos 7, 8, 14,17 e 21 dias após a imunização foram coletadas amostras de excretas de quatro aves por unidade experimental para contagem de oocistos de Eimeria sp. Foi coletado sangue de duas aves por unidade experimental aos 28 e 42 dias de idade para a avaliação do perfil eletroforético da imunoglubulina A (IgA). As amostras de soro foram submetidas a eletroforese vertical em gel de poliacrilamida a $12 \%$ na presença de agente redutor (SDSPAGE) (SILVA et al., 2008). As bandas protéicas reveladas foram, quantificadas por densitometria (Zênite Z-30 Turbo), comparando com um padrão de concentração gradiente de soro albumina bovina (MACIEL et al., 2007). Os soros das aves foram analisados individualmente em duplicata $\mathrm{e}$ os resultados foram as médias das densidades óticas determinadas em comprimento de onda de $495 \mathrm{~nm}$.

Estas mesmas aves foram sacrificadas e após retirada do intestino foi realizada avaliação de score das lesões em quatro regiões do intestino: duodeno e jejuno superior; intestino médio próximo ao divertículo. Baseado na severidade das lesões observadas, foi conferido à cada ave um score, com referência numa escala pré determinada de graus de lesão, onde: $0=$ lesão ausente $;+=$ separação entre o epitélio e a lâmina própria na extremidade das vilosidades; $++=$ exposição da lâmina própria na extremidade das vilosidades; $+++=$ exposição da lâmina própria de um terço até a metade da extensão das vilosidades; $++++=$ exposição da lâmina própria até a base das vilosidades foi registrado por cada ave sacrificada. 
Tabela 1. Composição percentual e calculada das dietas experimentais dos frangos de corte no período inicial ( 1 a 14 dias) e crescimento/final (15 a 42 dias)

\begin{tabular}{lcc}
\hline Ingredientes (\%) & Ração inicial & Ração cresc./final \\
\hline Milho & 53,02 & 59,97 \\
Farelo de Soja & 39,34 & 31,90 \\
Óleo de Soja & 3,15 & 4,11 \\
Calcário Calcítico & 0,98 & 1,00 \\
Fosfato Bicálcico & 2,09 & 1,60 \\
Caulim* & 0,15 & 0,15 \\
Sal Comum & 0,37 & 0,48 \\
Bicarbonato de Sódio & 0,09 & 0,00 \\
L-Lisina & 0,18 & 0,24 \\
DL-Metionina & 0,28 & 0,18 \\
L-Treonina & 0,04 & 0,07 \\
Premix vit. e mineral ${ }^{1,2}$ & 0,30 & 0,30 \\
\hline Valores calculados & & \\
\hline Proteína, \% & 22,50 & 19,00 \\
EM, Kcal/Kg & 2949 & 3100 \\
Cálcio, \% & 1,00 & 0,97 \\
Fósforo Disponível, \% & 0,50 & 0,47 \\
Sódio, \% & 0,19 & 0,19 \\
Potássio, \% & 0,87 & 0,72 \\
Cloro, \% & 0,30 & 0,30 \\
Lisina Dig., \% & 1,25 & 1,06 \\
Metionina Dig., \% & 0,59 & 0,49 \\
Met.+Cistina Dig., \% & 0,89 & 0,75 \\
Treonina Dig., \% & 0,80 & 0,68 \\
Arginina Dig., \% & 1,45 & 1,18 \\
Mongin, Meq/100g & 228,22 & 192,72 \\
\hline S Susito pela in &
\end{tabular}

* Substituído pela inclusão do produto a base de plantas ou Enramicina

${ }^{1}$ Mistura Vitamínica (Conteúdo por kg de premix): Vit. A 7.000.000,00 UI; Vit. D3 2.200.000,00 UI; Vit.E 11.000,00 mg; Vit. K3 1.600,00 mg; Vit. B1 2.000,00 mg; Vit. B2 5.000,00 mg, Vit. B12 12.000,00 mg; Niacina 35.000,00 mg; Ácido Pantotênico 13.000,00 mg; Ácido Fólico 800,00 mg; Antioxidante 100.000,00; Veículo q.s.p. 1.000,00g.

${ }^{2}$ Mistura mineral (Conteúdo por kg de premix): Ferro 10.000,00 mg; Cobre 16.000,00 mg; Iodo 2.400,00 mg; Zinco 100.000,00 mg; Manganês 140.000,00 mg; Selênio 400,00 mg; Veículo q.s.p. 1.000,00g.

Amostras da mucosa do intestino delgado foram coletadas para avaliação de $\operatorname{IgA}$, de acordo com a metodologia descrita para o perfil sérico de IgA. Amostras de segmento do duodeno, jejuno e íleo foram fixadas em solução de formol tamponado e incluídas em parafina para a obtenção de cortes transversais. As lâminas com cortes dos segmentos foram coradas pelo método de hematoxilina-eosina. As imagens do duodeno foram capturadas e analisadas no programa IMAGE PROPLUS 4.1.
Foi mensurada a altura de 20 vilos e a profundidade de 20 criptas de cada repetição por segmento e destes valores foi obtida a média. As pernas direita de cada ave foram devidamente identificadas e posteriormente avaliadas quanto ao grau de coloração. Foi utilizado o leque colorimétrico da Roche ${ }^{\circledR}$ e estabelecidos os scores de acordo com a intensidade da cor amarela. Os resultados obtidos foram submetidos à análise de variância utilizando $\mathrm{o}$ procedimento General Linear 
Model(GLM) do software SAS (SAS Institute, 2002) e as médias referentes às dietas foram comparadas a um nível de $5 \%$ de probabilidade pelo teste Tukey. Por não apresentarem distribuição normal, os dados referentes às lesões microscópicas da mucosa intestinal e coloração de perna foram analisados por teste não-paramétrico (Kruskal-Wallis).

\section{RESULTADOS}

Houve efeito linear decrescente $(p<0,05)$ para o desafio vacinal em função dos dias pós-inoculação sobre a eliminação de oocistos nas excretas das aves dos tratamentos que receberam a vacina oral contra coccidiose (Tabela 2 ).

De acordo com os resultados demonstrados na Tabela 3, aves desafiadas experimentalmente pela inoculação de oocistos de Eimeria $s p$ apresentaram títulos mais elevados de IgA $(\mathrm{p}<0,05)$ detectados na mucosa intestinal aos 28 dias de idade, independente do extrato de plantas adicionada à dieta. Entretanto, aos 42 dias de idade, o tratamento que recebeu desafio vacinal e foi suplementado com o extrato de plantas apresentou o maior título para $\operatorname{Ig} \mathrm{A}(\mathrm{p}<0,05)$.

Tabela 2. Contagem de oocistos de Eimeria sp (OOPG)

\begin{tabular}{lccccccc}
\hline Item & $7 \mathrm{dpi}^{*}$ & $8 \mathrm{dpi}$ & $14 \mathrm{dpi}$ & $17 \mathrm{dpi}$ & $21 \mathrm{dpi}$ & $\mathrm{CV}, \%$ & Efeito \\
\hline APC & 0,0 & 0,0 & 156,1 & 175,2 & 123,6 & 171,20 & $\mathrm{~ns}$ \\
APC + desafio vacinal & 1018,0 & 1350,7 & 67,6 & 228,7 & 122,8 & 191,04 & ${ }^{1}$ linear \\
Extrato plantas & 0,0 & 103,8 & 164,8 & 97,7 & 18,0 & 270,97 & ns \\
Extrato plantas + desafio vacinal & 1412,3 & 1733,5 & 119,9 & 277,5 & 318,7 & 130,00 & ${ }^{2}$ linear \\
\hline
\end{tabular}

Dpi = dias pós inoculação; ns: não significativo; ${ }^{1} \hat{\mathrm{Y}}=1429,405-302,158 \mathrm{x} ; \mathrm{R}^{2}: 0,65 ;{ }^{2} \hat{\mathrm{Y}}=1851,314-$ $371,119 x ; R^{2}: 0,80$.

Tabela 3. Perfil eletroforético da imunoglubulina IgA em soro e mucosa intestinal de frangos c corte suplementados com extrato de plantas e desafiados com Eimeria sp

\begin{tabular}{lcccc}
\hline \multirow{2}{*}{ Item } & \multicolumn{2}{c}{ Soro } & \multicolumn{2}{c}{ Mucosa intestinal } \\
\cline { 2 - 5 } & 28 dias & 42 dias & 28 dias & 42 dias \\
\hline APC & 0,206 & 0,283 & $0,343^{\mathrm{b}}$ & $0,318^{\mathrm{c}}$ \\
APC + desafio vacinal & 0,264 & 0,245 & $0,568^{\mathrm{a}}$ & $0,460^{\mathrm{b}}$ \\
Extrato plantas & 0,251 & 0,263 & $0,369^{\mathrm{b}}$ & $0,340^{\mathrm{c}}$ \\
Extrato plantas + desafio vacinal & 0,244 & 0,278 & $0,570^{\mathrm{a}}$ & $0,547^{\mathrm{a}}$ \\
CV\% & 32,54 & 25,38 & 22,73 & 18,77 \\
P & ns & ns & $*$ & $*$ \\
\hline
\end{tabular}

ns: não significativo, ${ }^{*} \mathrm{p}<0,05$

A avaliação do score de coloração das pernas dos frangos (Tabela 4) não mostrou diferenças significativas $(p>0,05)$ entre os tratamentos. Da mesma forma, a avaliação de lesões microscópicas na mucosa do duodeno, jejuno, íleo e ceco (Tabela 5) não mostrou diferenças significativas $(\mathrm{p}>0,05)$. 
Tabela 4. Score de coloração da perna sobre o percentual (\%) avaliado de frangos de corte suplementados com extrato de plantas e desafiados co Eimeria sp

\begin{tabular}{|c|c|c|c|c|c|c|c|c|}
\hline \multirow{3}{*}{ Item } & \multicolumn{8}{|c|}{ Coloração da perna (\%) } \\
\hline & \multicolumn{4}{|c|}{28 dias de idade } & \multicolumn{4}{|c|}{42 dias de idade } \\
\hline & $* 6$ & 7 & 8 & 9 & 6 & 7 & 8 & 9 \\
\hline APC & 27,7 & 33,3 & 22,2 & 11,1 & 0 & 0 & 44,4 & 55,5 \\
\hline $\mathrm{APC}+$ desafio vacinal & 50,0 & 27,7 & 16,6 & 5,55 & 0 & 0 & 55,5 & 44,4 \\
\hline Extrato plantas & 5,55 & 38,8 & 33,3 & 16,6 & 0 & 0 & 44,4 & 55,5 \\
\hline Extrato plantas + desafio vacinal & 44,4 & 33,3 & 16,6 & 5,55 & 0 & 0 & 22,2 & 77,7 \\
\hline Teste G & \multicolumn{4}{|c|}{11,67} & \multicolumn{4}{|c|}{2,27} \\
\hline $\mathrm{P}$ & \multicolumn{3}{|c|}{ ns } & & \multicolumn{4}{|c|}{ ns } \\
\hline
\end{tabular}

* Escala de cores (leque colorimétrico Roche $\left.{ }^{\circledR}\right)$.

Tabela 5. Score de lesão de mucosa de duodeno, jejuno, íleo e ceco sobre o percentual (\%) avaliado de frangos de corte suplementados com extrato de plantas e desafiados com Eimeria sp

\begin{tabular}{|c|c|c|c|c|c|c|c|c|c|c|c|c|c|c|c|c|c|c|c|c|}
\hline & \multicolumn{20}{|c|}{28 dias de idade } \\
\hline & \multicolumn{5}{|c|}{ Duodeno } & \multicolumn{5}{|c|}{ Jejuno } & \multicolumn{5}{|c|}{ Íleo } & \multicolumn{5}{|c|}{ Ceco } \\
\hline & $* 0$ & + & ++ & +++ & ++++ & 0 & + & ++ & +++ & ++++ & 0 & + & ++ & +++ & ++++ & 0 & + & ++ & +++ & ++++ \\
\hline APC & 11,1 & 33,3 & 27,7 & 27,7 & 0 & 33,3 & 38,8 & 22,2 & 5,55 & 0 & 94,4 & 5,55 & 0 & 0 & 0 & 83,3 & 11,1 & 5,55 & 0 & 0 \\
\hline $\mathrm{APC}+$ desafio & 11,1 & 55,5 & 33,3 & 0 & 0 & 33,3 & 61,1 & 0 & 5,55 & 0 & 94,4 & 5,55 & 0 & 0 & 0 & 83,3 & 16,6 & 0 & 0 & 0 \\
\hline E. plantas & 11,1 & 22,2 & 33,3 & 27,7 & 5,55 & 22,2 & 44,4 & 27,7 & 5,55 & 0 & 88,8 & 11,1 & 0 & 0 & 0 & 88,8 & 11,1 & 0 & 0 & 0 \\
\hline $\begin{array}{l}\text { E. plantas + } \\
\text { desafio }\end{array}$ & 33,3 & 33,3 & 27,7 & 5,55 & 0 & 27,7 & 38,8 & 33,3 & 0 & 0 & 72,2 & 27,8 & 0 & 0 & 0 & 77,7 & 22,2 & 0 & 0 & 0 \\
\hline Teste G & \multicolumn{5}{|c|}{19,15} & \multicolumn{5}{|c|}{12,53} & \multicolumn{5}{|c|}{4,97} & \multicolumn{5}{|c|}{3,87} \\
\hline \multirow[t]{4}{*}{ Valor de $\mathrm{p}$} & \multicolumn{5}{|c|}{ ns } & \multicolumn{5}{|c|}{ ns } & \multicolumn{5}{|c|}{ ns } & \multicolumn{5}{|c|}{ ns } \\
\hline & \multicolumn{20}{|c|}{42 dias de idade } \\
\hline & \multicolumn{5}{|c|}{ Duodeno } & \multicolumn{5}{|c|}{ Jejuno } & \multicolumn{5}{|c|}{ Íleo } & \multicolumn{5}{|c|}{ Ceco } \\
\hline & 0 & + & ++ & +++ & ++++ & 0 & + & ++ & +++ & ++++ & 0 & + & ++ & +++ & ++++ & 0 & + & ++ & +++ & ++++ \\
\hline APC & 44,4 & 11,1 & 44,4 & 0 & 0 & 33,3 & 33,3 & 33,3 & 0 & 0 & 88,8 & 11,1 & 0 & 0 & 0 & 100 & 0 & 0 & 0 & 0 \\
\hline $\mathrm{APC}+$ desafio & 44,4 & 33,3 & 22,2 & 0 & 0 & 11,1 & 33,3 & 44,4 & 0 & 11,1 & 66,6 & 22,2 & 11,1 & 0 & 0 & 77,7 & 11,1 & 11,1 & 0 & 0 \\
\hline E. plantas & 11,1 & 22,2 & 66,6 & 0 & 0 & 11,1 & 11,1 & 77,7 & 0 & 0 & 44,4 & 22,2 & 33,3 & 0 & 0 & 100 & 0 & 0 & 0 & 0 \\
\hline $\begin{array}{l}\text { E. plantas + } \\
\text { desafio }\end{array}$ & 22,2 & 22,2 & 55,5 & 0 & 0 & 22,2 & 22,2 & 44,4 & 0 & 11,1 & 55,5 & 22,2 & 22,2 & 0 & 0 & 100 & 0 & 0 & 0 & 0 \\
\hline Teste G & \multicolumn{5}{|c|}{6,07} & \multirow{2}{*}{\multicolumn{5}{|c|}{$\begin{array}{c}7,52 \\
\text { ns }\end{array}$}} & \multicolumn{5}{|c|}{6,47} & \multicolumn{5}{|c|}{5,91} \\
\hline $\mathrm{P}$ & \multicolumn{5}{|c|}{ ns } & & & & & & \multicolumn{5}{|c|}{ ns } & \multicolumn{5}{|c|}{$\mathrm{ns}$} \\
\hline
\end{tabular}

*Score| 0: ausente, +: separação entre o epitélio e a lâmina própria na extremidade das vilosidades, ++: exposição da lâmin
própria de um terço até a metade da extensão das vilosidades, ++++: exposição da lâmina própria até a base das vilosidades. 
$\mathrm{Na}$ Tabela 6 estão demonstrados os resultados zootécnicos. As aves que sofreram desafio vacinal, apresentaram menor ganho de peso $(p<0,05)$ na semana seguinte ao desafio em comparação às aves dos tratamentos que não receberam a vacina.

A conversão alimentar foi pior $(p<0,05)$ para as aves dos tratamentos a base de extrato de plantas. O peso vivo das aves do tratamento que recebeu o extrato de plantas e foi desafiado ainda se manteve menor $(\mathrm{p}<0,05)$ aos 28 dias de idade, entretanto, o ganho de peso e a conversão alimentar foram semelhantes para todos os tratamentos (Tabela 6).

Tabela 6. Desempenho produtivo de frangos de corte suplementados com extrato de plantas e desafiados com Eimeria sp

\begin{tabular}{lcccc}
\hline Tratamentos & $\begin{array}{c}\text { Peso } \\
\text { vivo,g }\end{array}$ & $\begin{array}{c}\text { Ganho de } \\
\text { peso,g }\end{array}$ & $\begin{array}{c}\text { Consumo } \\
\text { de ração,g }\end{array}$ & $\begin{array}{c}\text { Conversão } \\
\text { alimentar }\end{array}$ \\
\hline APC & $21 \mathrm{~d}$ & & $14-21 \mathrm{~d}$ & \\
APC + desafio vacinal & 1080,77 & $602,60^{\mathrm{a}}$ & $751,19^{\mathrm{a}}$ & $1,248^{\mathrm{b}}$ \\
Extrato plantas & 997,76 & $520,36^{\mathrm{b}}$ & $682,54^{\mathrm{b}}$ & $1,322^{\mathrm{ab}}$ \\
Extrato plantas + desafio & 1041,70 & $566,28^{\mathrm{a}}$ & $796,61^{\mathrm{a}}$ & $1,408^{\mathrm{a}}$ \\
vacinal & 983,79 & $505,25^{\mathrm{b}}$ & $723,49^{\mathrm{ba}}$ & $1,414^{\mathrm{a}}$ \\
CV,\% & 5,30 & 5,71 & 6,80 & 8,15 \\
$\mathrm{P}$ & $\mathrm{ns}$ & $*$ & $*$ & $*$ \\
\hline & $28 \mathrm{~d}$ & & $21-28 \mathrm{~d}$ & \\
\hline APC & $1648,62^{\mathrm{a}}$ & 561,95 & 884,14 & 1,575 \\
APC + desafio vacinal & $1568,88^{\mathrm{ab}}$ & 565,49 & 810,86 & 1,614 \\
Extrato de plantas & $1585,65^{\mathrm{ab}}$ & 532,57 & 770,36 & 1,558 \\
Extrato de plantas + desafio & $1505,04^{\mathrm{b}}$ & 521,25 & 829,24 & 1,704 \\
vacinal & 5,83 & 14,74 & 12,34 & 8,20 \\
CV,\% & $*$ & $\mathrm{~ns}$ & $\mathrm{~ns}$ & $\mathrm{~ns}$ \\
P & $42 \mathrm{~d}$ & & $1-42 \mathrm{~d}$ & \\
\hline & 2763,33 & 2710,09 & 4733,75 & 1,747 \\
\hline APC & 2678,69 & 2625,93 & 4688,79 & 1,786 \\
APC + desafio vacinal & 2680,51 & 2627,39 & 4742,68 & 1,805 \\
Extrato de plantas & 2749,49 & 2695,03 & 4788,70 & 1,777 \\
Extrato de plantas + desafio & 4,54 & 6,22 & 5,79 & 5,99 \\
vacinal & $\mathrm{ns}$ & $\mathrm{ns}$ & $\mathrm{ns}$ & $\mathrm{ns}$ \\
CV,\% & & & & \\
P & & &
\end{tabular}

Ns $=$ não significativo, ${ }^{*} \mathrm{p}<0,05$

Os resultados referentes a morfometria da mucosa intestinal (comprimento do vilo, profundidade das criptas e relação vilo: cripta) aos 28 dias estão apresentados na Tabela 7. Houve diferença significativa $(\mathrm{p}<0,05)$ apenas no comprimento do vilo e na relação vilo:cripta do duodeno dos frangos aos
28 dias de idade. As aves do tratamento controle apresentaram vilos maiores apenas em relação às aves do tratamento que recebeu extrato de plantas, enquanto houve uma menor relação vilo:cripta das aves deste tratamento em relação às aves que receberam a vacina. Pode ser observado, que, apesar de não 
significativa, a profundidade das criptas foram menores em aves vacinadas, 0 que contribuiu para uma maior relação vilo:cripta. Aos 42 dias de idade, a análise morfométrica da mucosa do intestino delgado não apontou diferenças significativas $(\mathrm{p}>0,05)$

Tabela 7. Comprimento de vilo, profundidade de cripta e relação vilo:cripta (V:C) do duodeno, jejuno e íleo de frangos de corte aos 28 e 42 dias de idade suplementados com extrato de plantas e desafiados com Eimeria sp

\begin{tabular}{|c|c|c|c|c|c|c|c|c|c|}
\hline \multirow[b]{2}{*}{ Item } & \multicolumn{3}{|c|}{ Duodeno } & \multicolumn{3}{|c|}{ Jejuno } & \multicolumn{3}{|c|}{ Íleo } \\
\hline & $\begin{array}{l}\text { Vilo, } \\
\mu \mathrm{m}\end{array}$ & $\begin{array}{c}\text { Cripta } \\
\mu \mathrm{m}\end{array}$ & $\mathrm{V}: \mathrm{C}$ & $\begin{array}{l}\text { Vilo, } \\
\mu \mathrm{m}\end{array}$ & $\begin{array}{c}\text { Cripta } \\
\mu \mathrm{m}\end{array}$ & $\mathrm{V}: \mathrm{C}$ & $\begin{array}{l}\text { Vilo, } \\
\mu \mathrm{m}\end{array}$ & $\begin{array}{c}\text { Cripta } \\
\mu \mathrm{m}\end{array}$ & $\mathrm{V}: \mathrm{C}$ \\
\hline & \multicolumn{9}{|c|}{28 dias } \\
\hline APC & $551,61^{\mathrm{a}}$ & 51,41 & $11,03^{\mathrm{ba}}$ & 455,67 & 57,91 & 8,13 & 286,71 & 41,51 & 7,15 \\
\hline $\begin{array}{l}\text { APC + } \\
\text { desafio }\end{array}$ & $526,32^{\text {ba }}$ & 44,89 & $12,36^{\mathrm{a}}$ & 429.07 & 53,21 & 8,09 & 324,28 & 43,51 & 7,75 \\
\hline E. plantas & $460,44^{\mathrm{b}}$ & 53,53 & $9,020^{\mathrm{b}}$ & 420,44 & 57,58 & 7,48 & 297,52 & 43,12 & 7,12 \\
\hline $\begin{array}{l}\text { E. plantas } \\
+ \text { desafio }\end{array}$ & $504,72^{\text {ba }}$ & 50,87 & $10,92^{\text {ba }}$ & 446,54 & 51,55 & 8,68 & 308,62 & 41,39 & 7,70 \\
\hline $\mathrm{CV}, \%$ & 13,20 & 28,38 & 27,43 & 13,16 & 17,68 & 14,92 & 18,76 & 22,19 & 25,35 \\
\hline \multirow[t]{2}{*}{$P$} & $*$ & ns & $*$ & $\mathrm{~ns}$ & ns & $\mathrm{ns}$ & ns & ns & ns \\
\hline & \multicolumn{9}{|c|}{42 dias } \\
\hline APC & 895,26 & 75,64 & 11,84 & 732,77 & 83,61 & 8,76 & 448,94 & 55,94 & 8,03 \\
\hline $\begin{array}{l}\text { APC + } \\
\text { desafio }\end{array}$ & 946,76 & 90,62 & 10,45 & 709,01 & 80,46 & 8,81 & 429,81 & 56,52 & 7,60 \\
\hline E. plantas & 928,30 & 81,38 & 11,41 & 707,12 & 86,67 & 8,16 & 473,34 & 53,13 & 8,91 \\
\hline $\begin{array}{l}\text { E. plantas } \\
+ \text { desafio }\end{array}$ & 881,68 & 80,40 & 10,97 & 747,72 & 86,75 & 8,62 & 467,30 & 57,69 & 8,10 \\
\hline $\mathrm{CV}, \%$ & 17,09 & 22,91 & 27,80 & 16,27 & 14,34 & 20,80 & 20,34 & 18,38 & 30,44 \\
\hline $\mathrm{P}$ & ns & ns & ns & ns & $\mathrm{ns}$ & ns & ns & ns & $\mathrm{ns}$ \\
\hline
\end{tabular}

Ns $=$ não significativo, ${ }^{*} \mathrm{p}<0,05$.

\section{DISCUSSÃO}

A eliminação decrescente de oocistos de Eimeria nas excretas das aves dos tratamentos que receberam o desafio vacinal demonstra a eficiência do desafio experimental e indica que a redução significativa do número de oocistos nas unidades, com aplicação da vacina se deve, provavelmente ao estímulo do sistema imune pelos antígenos vacinais e a proteção conferida aos enterócitos, à multiplicação e eliminação dos oocistos de Eimeria.

A inclusão de extratos de plantas na dieta das aves desafiadas com a vacina de coccidiose resultou em maiores títulos para IgA na mucosa intestinal dos frangos de corte aos 42 dias de idade. Este resultado demonstra que o extrato de plantas utilizado pode ter efeito estimulatório sobre o sistema de defesa local da mucosa intestinal. O cardanol, princípio ativo presente no caju (Anacardium occidentale L.) e que compõe o produto comercial utilizado neste experimento, tem atividade tanto anti-inflamatória como antioxidante (TREVISAN et al., 2006), o que pode ter contribuído com este resultado.

A maior superfície de contato do organismo com o meio externo é a mucosa que recobre $o$ trato gastrointestinal. Além da mucosa gastrointestinal constituir-se numa porta 
de entrada aos antígenos alimentares e às bactérias não patogênicas da microflora, representa uma forte fonte de perturbação da atividade imunológica no organismo. É também na mucosa que se localiza o maior número de macrófagos, polimorfonucleares, células dendrídicas, linfócitos $\mathrm{T}$ e linfócitos $\mathrm{B}$, secretores de imunoglobulinas (FRIEDMAN et al., 2003).

As lesões microscópicas na mucosa do duodeno, jejuno, íleo e ceco não difereriram entre aves desafiadas ou suplementadas com extratos de plantas. Resultado que se contrapõe ao trabalho realizado por Bona et al. (2012) que mostrou que o uso de avilamicina e o composto vegetal a base de óleos essenciais na dieta reduziram significativamente os escores de lesões intestinais causados por E. maxima e $E$. tenella aos 29 dias de idade (14 dias após a inoculação). É importante considerar que a atividade antimicrobiana, antioxidante ou imunomoduladora pode variar de acordo com a planta utilizada.

Assim de acordo com a concentração do princípio ativo principal, os extratos vegetais possuem uma ou mais atividades biológicas, tais como ação antiviral, antifúngica, antimicrobiana, anticoccidiana (CHRISTAKI et al., 2004; YOUN \& NOH, 2001), além da pródigestiva e antioxidante (CEYLAN \& FUNG, 2004). Christaki et al. (2004) observaram uma redução nas lesões em ceco dos animais tratados com mistura comercial de óleos essenciais em frangos desafiados com Eimeria tenella. Bona et al. (2012) ainda concluiram que o uso de um composto vegetal e a avilamicina foram capazes de reduzir significativamente as enterites específicas causadas por Eimeria maxima e $E$. tenella nas aves aos 29 dias de idade (14 dias após a inoculação), e podem ser utilizados no controle destas enfermidades.
O menor ganho de peso das aves desafiadas observado na semana seguinte à inoculação, pode ser explicado pela demanda de nutrientes para manter a homeostase frente ao desafio imunológico e reparação da mucosa agredida pela replicação das eimérias. $\mathrm{O}$ uso do aditivo fitogênico não evitou a perda de peso das aves desafiadas com a vacina. Quando a mucosa sofre processo de agressão, a reposição celular se faz às custas de consumo de nutrientes, os quais são provenientes das reservas energéticas do organismo da ave e da ração ingerida. McBride \& Kelly (1990) estimaram que a manutenção do epitélio intestinal e estruturas anexas de suporte tem custo de $20 \%$ da energia bruta consumida pelo animal.

A pior conversão alimentar observada para as aves que foram suplementadas com extrato de plantas na primeira semana pós-infecção pode ser devido ao mecanismo de recuperação dos aditivos fitogênicos em comparação aos melhoradores do desempenho, uma vez que o consumo de ração foi semelhante. Neste trabalho, observou-se uma pior conversão alimentar com o uso dos extratos de plantas e com um menor comprimento de vilo apenas uma semana pós infecção. Apesar da maior demora na recuperação da integridade funcional da mucosa intestinal, os dados obtidos na idade de abate são semelhantes entre a dieta controle, que continha melhorador do desempenho e a dieta composta por extrato de plantas. Christaki et al. (2004) e Koiyama et al. (2014) concluíram que aditivos fitogênicos podem proporcionar desempenho zootécnico comparáveis ao do uso de antibióticos melhoradores do desempenho.

A literatura confirma ainda efeitos positivos de adtivos fitogênicos sobre a digestibilidade e algumas características histológicas e morfométricas do intestino 
(IBRIR et al., 2002; MANZANILLA et al., 2004; FUKAYAMA et al., 2005).

A mamona (Ricinus communis L.), que compõe o extrato utilizado, contém $90 \%$ de ácido graxo ricinoléico, o qual confere ao óleo suas características singulares. Já o caju possui como princípios ativos oriundos do seu óleo o ácido anacárdico $(2-3 \%)$, cardol $(15-18 \%)$ e cardanol (75 - 80\%). Apesar da utilização de plantas com fins medicinais para tratamento cura e prevenção de doenças, pode ser uma das mais antigas formas de prática medicinal da humanidade (VEIGA JUNIOR \& PINTO, 2005), o uso de extratos vegetais como promotores de crescimento em animais ainda é um assunto recente, com o número de pesquisas aumentando gradativamente devido a diversos fatores, como por exemplo, a resposta espécie-específica, a toxicidade e o efeito da associação de princípios ativos diversos (APPLEGATE et al., 2010).

Alguns trabalhos têm relatado uma redução na palatabilidade da dieta através da inclusão de aditivos fitogênicos (SCHÖNE et al., 2006). O uso de alguns extratos de plantas pode resultar em odores fortes ou até mesmo um sabor acentuado nas rações. Por outro lado, há registros de melhora no consumo de ração (COSTA et al., 2007), uma vez que os fitogênicos, em doses menores, influenciam o flavour e a palatabilidade, sendo um atrativo a mais para o maior consumo de ração.

O uso dos fitogênicos na alimentação animal está restrito a diversas normas e normalmente são considerados como produtos adicionados à ração de animais saudáveis com função nutricional durante o período de criação, ao contrário dos medicamentos veterinários (APPLEGATE et al., 2010), os quais são utilizados em animais com problemas de saúde por um tempo determinado.
Barreto et al. (2008) não observaram diferença significativa no desempenho de frangos de corte frente ao uso de óleos essenciais de orégano, cravo, canela e pimenta vermelha. Fukayama et al. (2005) encontraram características semelhantes entre aves suplementadas com antibiótico e extratos de plantas quanto ao desempenho, à qualidade da carcaça, à avaliação anátomo-fisiológica do trato digestório $\mathrm{e}$ às bactérias encontradas no ceco das aves. Toledo et al. (2007) também observaram desempenho produtivo semelhante entre aves tratadas com aditivos fitogênicos e antibiótico promotor de crescimento.

É importante ressaltar, que estes resultados foram obtidos em condições experimentais. Desta forma, o uso de aditivos fitogênicos podem não ter $o$ mesmo impacto quanto utilizado nas dietas para frangos de corte criados em condições comerciais. A reduzida densidade de criação, a utilização de cama nova a cada lote, os procedimentos de desinfecção e a falta de desafio sanitário ou ambiental contribuem sobremaneira na manutenção do estado de saúde e no desempenho produtivo das aves. Embora alguns efeitos positivos dos aditivos fitogênicos já tenham sido demonstrados mesmo em criações comerciais, há ainda grande desconhecimento dos mecanismos de ação destes produtos, o que influencia nos resultados e eficiência das diversas pesquisas realizadas nesta área (KOIYAMA et al., 2014).

Estudos da toxicidade relacionados a dose, interação de princípios ativos e relação com a microbiota intestinal são necessários ainda para assegurar a melhor forma de utilizá-los (APPLEGATE et al., 2010). Outro aspecto importante que deve ser explorado é a utilização de fitogênicos associado à inclusão de outros aditivos naturais nas dietas, de forma a investigar melhor os possíveis efeitos sinergéticos 
promovidos pelo uso de compostos associados nas rações. Por fim, o uso de extratos de plantas em substituição aos melhoradores de desempenho alia-se a necessidade de redução de custos e perdas na produção de frangos de corte, diante também de um mercado consumidor cada vez mais exigente quanto a forma de criação dos animais e a qualidade dos produtos.

Os resultados obtidos mostraram que a adição de extrato de plantas à dieta de aves desafiadas com oocistos de Eimeria $s p$ aumentou a secreção de IgA pela mucosa intestinal. O desafio com oocistos de Eimeria $s p$ ou associado ao extrato de plantas resultou em menor ganho de peso e pior conversão alimentar apenas na semana seguinte à inoculação.

A inclusão do extrato de plantas ou de melhorador de desempenho resultou em desempenho produtivo semelhante na idade de abate.

\section{REFERÊNCIAS}

APPLEGATE, T.J.; KLOSE, V.; STEINER, T.; GANNER, A.; SCHATZMAYR, G. Probiotics and phytogenics for poultry: Myth or reality? Journal of Applied Poultry

Research, v.19, n.2, p.194-210, 2010.

BARRETO, M.S.R.; MENTEN, J.F.M.; RACANICCI, A.M.C.; PEREIRA, P.W.Z.; RIZZO, P.V. Plant extracts used as growth promoters in broilers. Brazilian Journal of Poultry Science, v.10, n.2, p.109-115, 2008.

BONA, T.D.M.M.; PICKLER, L.; MIGLINO, L.B.; Kuritza, L.N.; VASCONCELOS, S.P.; SANTIN, E. Óleo essencial de orégano, alecrim, canela e extrato de pimenta no controle de Salmonella, Eimeria e Clostridium em frangos de corte. Pesquisa

Veterinária Brasileira, v.32, n.5, p.411-418, 2012.

CEYLAN, E.; FUNG, D.Y.C. Antimicrobial activity of spices. Journal of Rapid Methods and Automation in Microbiology, v.12, n.1, p.1-55, 2004.

CHRISTAKI, E.; FLOROU-PANERI, P.; GIANNENAS, I. Effect of a mixture of herbal extracts on broiler chickens infected with Eimeria tenella. Animal Resources, v.53, n.2, p.137-144, 2004.

COSTA, L.B.; TSE, M.L.P.; MIYADA, Y.S. Extratos vegetais como alternativas aos antimicrobianos promotores de crescimento para leitões recémdesmamados. Revista Brasileira de Zootecnia, v.36, n.3, p.589-595, 2007.

FRIEDMAN, A.; BAR-SHIRA, E.; SKLAN, D. Ontogeny of gut associated immune competence in the chick.

World's Poultry Science Journal, v.59, n.2, p.209-219, 2003.

FUKAYAMA, E.H.; BERTECHINI, A.G.; GERALDO, A. et al. Extrato de Orégano como Aditivo em Rações para Frangos de Corte. Revista Brasileira de Zootecnia, v.34, n.6, p.2316-2326, 2005.

HUYGHEBAERT, G.; DUCATELLE, R.; VAN IMMERSEEL, F. An update on alternatives to antimicrobial growth promoters for broilers. The Veterinary

Journal, v.187, n.2, p.182-188, 2011.

IBRIR, F.; GREATHEAD, H.M.R.; FORBES, J.M. The effect of thymol/carvacrol treatments on performance of broiler chickens infected with Eimeria acervulina. In: THE NUTRITION SOCIETY, 2002, London. Proceedings.... London, 2002. p.61. 
JAMROZ, D.; WERTELECKI, T.; HOUSZKA, M.; KAMEL, C. Influence of diet type on the inclusion of plant origin active substances on morphological and histochemical characteristics of the stomach and jejunum walls in chicken.

Journal of Animal Physiology and Animal Nutrition, v.90, n.3, p.255-268, 2006.

JEAUROUND, E.; SCHUMANN, B.; CLUNIES, M. Supplementation of diets with herbal extracts enhances growth performance in newly-weaned piglets. Journal of Animal Science, v.20, p.394, 2002. Suppl.1.

KOIYAMA, N.T.G; ROSA, A.P; PADILHA, M.T.S.; BOEMO, L.S.; SCHER, A.; MELO, A.M.S.; FERNANDES, M.O. Desempenho e rendimento de carcaça de frangos de corte alimentados com mistura de aditivos fitogênicos na dieta. Pesquisa

Agropecuária Brasileira, v.49, n.3, p.225-231, 2014.

MACIEL, R.M.; LOPES, S.T.A.; SANTURIO, J.M.; ROSA, A.P.; DUARTE, M.M.M.F.; MARTINS, D.B.; EMANUELLI, M.P. Perfil eletroforético das proteínas séricas de frangos de corte alimentados com dietas contendo aflatoxinas e/ou argila clinoptilolita natural. Ciência Rural, v.37, n.3, p.744749, 2007.

MANZANILLA, E.G.; PEREZ, J.F.; MARTIN, M.; KAMEL, C.; BAUCELLS, F.; GASA, J. Effect of plant extracts and formic acid on intestinal equilibrium of early-weaned pigs. Journal of Animal Science, v.82, n.11, p.3210-3218, 2004.
MCBRIDE, B.W.; KELLY, J.M. Energy cost of absorption and metabolism in the ruminant gastrointestinal tract and liver: a review. Journal of Animal Science, v.68, n.9, p.2997-3010, 1990.

McDEVITT, R.M.; BROOKER, J.D.; ACAMOVIC, T.; SPARKS, N.H.C. Necrotic enteritis; a continuing challenge for the poultry industry. World's Poultry Science Journal, v.62, n.2, p.221-247, 2006.

PEARCE, M.; JIN, G.L.Z. Aditivos Fitogênicos. Porkworld, v.58, p.128-136, 2010.

RIZZO P.V.; MENTEN, J.F.M.; RACANICCI, A.M.C.; TRALDI, A.B.; SILVA, C.S.; PEREIRA, P.W.Z. Extratos vegetais em dietas para frangos de corte. Revista Brasileira de Zootecnia, v.39, n.4, p.801-807, 2010.

SCHÖNE, F.; VETTER, A.; HARTUNG, H.; BERGMANN, H.; BIERTÜMPFEL, A.; RICHTER, G.; MÜLLER, S.; BREITSCHUH, G. Effects of essential oils from fennel (Foeniculi aetheroleum) and caraway (Carvi aetheroleum) um pigs. Journal of Animal Physiology and Animal Nutrition, v.90, n.11-12, p.500-510, 2006.

SILVA, R.O.P.; LOPES, A.F.; FARIA, R.M.D. Eletroforese de proteínas séricas: interpretação e correlação clínica. Revista Médica de Minas Gerais, v.18, n.2, p.116-22, 2008.

TOLEDO, G.S.P.; COSTA, P.T.C.; SILVA L.P. Desempenho de frangos de corte alimentados com dietas contendo antibiótico e/ou fitoterápico como promotores, adicionados isoladamente ou associados. Ciência Rural, v.37, n.6, p.1760-1764, 2007. 
TREVISAN M.T.S., PFUNDSTEIN B., HAUBNER R. Characterization of alkyl phenols in cashew (Anacardium occidentale) products and assay of their antioxidant capacity. Food Chemical Toxicology, v. 44, n. 2, p.188-97, 2006.

VEIGA JUNIOR V.P, PINTO, A.C. Plantas Medicinais : Cura segura? Química Nova, v.28, n.3, p.519-528, 2005.

YOUN, H.J.; NORTH, J.W. Screening of the anticoccidial effects of herb extracts against Eimeria tenella. Veterinary

Parasitology, v.96, n.4, p.257-263, 2001.

Data de recebimento: 24/05/2016

Data de aprovação: 24/10/2016 